Tobacco Control

\section{Entering our 16th year}

Simon Chapman

\section{6th year of Tobacco control}

W ith this issue, Tobacco Control enters its 16th year. The journal is in robust health, with its web traffic rising exponentially, thanks largely to the huge success of our publisher's venture in selling the journal to libraries and institutions as part of a bundled package of online accessible journals in the $B M J$ stable. In the last week of December 2005, viewers opened 36521 articles. Just one paper, on the health consequences of reducing cigarette consumption, ${ }^{1}$ has been opened 8118 times since early December. Our most cited paper $^{2}$ has been opened 25644 times since 1999. Each issue sees a paper producing headlines in global news media. The important association that exists between news publicity, web hits and subsequent citations ${ }^{3}$ is hopefully a sign of even further impact: the influence of research that we publish on policy, practice and, ultimately, on tobacco use.

\section{REJECTING PAPERS}

One of the most onerous tasks editors face is rejecting papers. In our lives as authors, the journal's five senior editors all know the disappointment of rejection. Authors always feel their papers deserve publication, but they lack the perspective of editors who must continuously consider the relative merits of each paper against all others concurrently being submitted.

The commercial reality of publishing, which authors often fail to appreciate, is that there are costs involved in publishing each paper, which must be met by sales of the journal to individual or institutional subscribers. The income received allows the publishers to set a budget for the number of pages that can be published (because the continual availability of the journal in paper form remains the single biggest constraint on our ability to publish more papers). In 2006, we published 488 pages across six issues. After allowing for our news, editorial and lighter side commitments, we were able to publish 63 original articles and reviews, six brief reports, four industry or ad watch commentaries, 13 letters and one abstract (with the full article online): a total of 87 articles, boosted by 42 papers published in four commissioned supplements. In 2006, we made editorial decisions on 388 papers. We accepted $135(34.8 \%)$ and rejected 253.

\section{TRIAGING SYSTEM}

Knowing that the constraints on space described above necessitate us rejecting many papers, we have for several years operated an editorial "triaging" system where we reject papers without review. In 2006, 210 (54.1\% of all submissions) were thus rejected. It is a truism that peer reviewed journals could not be published without the help of peer reviewers. In 2006, 206 individuals freely gave their valuable time to review papers for us (see http://tc.bmj.com/misc/reviewers.dtl). ${ }^{3}$ It is our impression that securing reviewers is becoming increasingly difficult. One paper this year attracted 12 consecutive reviewer refusals before we secured even one review. Against this background, we do not wish to burden reviewers often with hours of effort when we judge that a paper is likely to be of less interest or importance to the broad, international field of population-oriented tobacco control policy and strategy than other papers with which it is competing for space.

Occasionally, authors are angered by such rejection. One said that editors should leave the entire matter of editorial judgement to reviewers, implying that all papers judged as being of publishable standard by reviewers should be accepted. Such a prescription would soon cause a log jam of accepted but unpublished papers, which would anger authors wanting to see their papers published expeditiously. Here, online only publication can appear an attractive option, bypassing expensive printing and mailing costs. But online papers still absorb substantial copyediting and online publication costs. Moreover, converting the journal to an online only journal to allow more papers to be published would subvert the still critical lifeline of paper journal subscribers, whose subscriptions would disappear, undermining the commercial viability of the journal.

This means that for now, we are committed to fitting the number of accepted articles into the paper space permitted by the economics of the journal. This commercial reality brings considerable advantages in forcing us to consider what sort of papers we want to publish rather than being slaves to more narrowly defined judgement about only the methodological standards of a paper. The editors give a lot of thought to the sort of journal we want Tobacco Control to be. Broadly speaking, the most important test that papers must pass before being selected to be sent out for peer review is whether we feel that they say anything important that is likely to attract substantial international reader interest; whether they can successfully and convincingly deal with the required question of "what this paper adds"; and whether the paper carries any message that is likely to add another clear, original "brick in the wall" of accumulating knowledge about tobacco use and its control.

\section{THE “INTERNATIONAL" TEST}

Passing the "international" interest test can be challenging. Local smoking prevalence studies and those analysing the antecedents and correlates of uptake, cessation or use of services will generally be of little interest to readers outside the province or country in question and so are more suited to regional or national journals. But occasionally such studies become more interesting because of other issues described in the paper, such as the influence of economic transition on consumption or the effect of a unique policy initiative.

Some of the most common reactions our senior editors make about papers they recommend for rejection without review are: we know this already; I can't imagine many people wanting to read this; it nearly put me to sleep; or that it describes an intervention which has little-to-no potential for population-wide impact. There are only so many times we need confirmatory studies showing that indoor air quality improves when smoking is banned, that smokers cost governments lots of healthcare dollars, or that quit and win cessation contests attract many entrants.

Above all, we try to make every issue of Tobacco Control something that, like a quality magazine or anthology, provides compelling reading that will cause readers to mentally bookmark papers for use in further research, policy advocacy and programme development.

Tobacco Control 2007; 16:1.

doi: 10.1136/tc. 2006.019802

Correspondence to: Professor S Chapman School of Public Health, Edward Ford Building A27, University of Sydney, NSW 2006, Sydney, Australia; sc@med.usyd.edu.au

Competing interests: None declared.

\section{REFERENCES}

1 Tverdal A, Bjartveit K. Health consequences of reduced daily cigarette consumption. Tob Control 2006;15:472-80.

2 Tyas SL, Pederson LL. Psychosocial factors related to adolescent smoking: a critical review of the literature. Tob Control 1998;7:409-20.

3 Chapman S, Nguyen TN, White C. Press released papers are more downloaded and cited. Tob Control 2007;16:71 